\title{
Forças em capacitores assimétricos submetidos a altas tensões
}

(Strengths on asymmetric capacitors under high voltage)

\author{
Carlos Alberto Kuhnen ${ }^{1}$, Eduardo de Almeida Isoppo e Gerson Renzetti Ouriques \\ Departamento de Física, Universidade Federal de Santa Catarina, Florianópolis, SC, Brasil \\ Recebido em 29/11/2006; Aceito em 1/3/2007
}

\begin{abstract}
Este trabalho trata de forças que atuam em capacitores assimétricos quando são utilizadas fontes de alta tensão. Estas forças são as responsáveis pelo fenômeno conhecido por "efeito Biefeld-Brown" que se acreditava ser um novo princípio físico envolvendo eletromagnetismo e gravitação. Capacitor assimétrico é um capacitor cujos eletrodos possuem geometria bastante diferenciada um do outro. Os resultados experimentais mostram que o capacitor, quando sob alta tensão, fica submetido à ação de uma força resultante produzida pela colisão de íons formados no ar (vento iônico) em volta dos eletrodos. A força resultante produz o chamado 'efeito levitação' que faz o capacitor flutuar em pleno ar. O sistema desenvolvido tem sido utilizado em demonstrações em sala de aula para estudantes dos cursos de graduação e pós-graduação em física da UFSC e para colegas do departamento de física, provocando grande excitação e discussões sobre o assunto.
\end{abstract}

Palavras-chave: capacitor assimétrico, alta-tensão, eletromagnetismo, íons, vento iônico.

This work deals with electrical forces on asymmetric capacitors when a source of high voltage is used. These strengths are responsible by the phenomenon known as "Biefeld-Brown effect" believed to be a new physical principle due to the electromagnetism and gravitation. Asymmetric capacitor is a capacitor whose geometry of the electrodes differs from each other. The experimental results show that when a high voltage is applied to the capacitor a resulting force acts on it, which is produced by the collision of the ions that are formed in the air (ionic wind) at vicinities of the electrodes. This resulting force promotes the so called "levitation effect" that makes the capacitor floating in the air. The system developed here have been used in classroom demonstrations for undergraduate and graduate students of the Federal University of Santa Catarina and for colleagues of the physics department provoking great excitement and discussions on the subject.

Keywords: asymmetric capacitor, high voltage, electromagnetism, ions, ionic wind.

\section{Introdução}

Em experimentos realizados com válvulas de raios-X na década de 1920, Thomas Townsend Brown (1905-1985) descobriu uma força resultante atuando na válvula quando esta era ligada. Desde então este fenômeno ficou conhecido como efeito Biefeld-Brown e acreditouse tratar de um novo princípio do eletromagnetismo e gravidade. Esta descoberta é creditada a Brown, porém leva também o nome de seu orientador Paul Alfred Biefeld (1867-1940), professor de física na Universidade de Denison, Ohio, na América do Norte.

A descoberta de Brown mostra que quando uma alta voltagem $(\sim 1 \mathrm{kV}$ DC) é aplicada em um capacitor assimétrico, uma força resultante na direção de um dos eletrodos é observada. Por capacitor assimétrico quer se dizer um capacitor cujas dimensões físicas de seus eletrodos são bastante diferentes. Por exemplo, um com grande área (uma placa metálica) e outro com área

\footnotetext{
${ }^{1}$ E-mail: kuhnen@fsc.ufsc.br.

muito pequena (um fio). Segundo Brown o fenômeno se dá no vácuo, porém quando nas condições ambientes acredita-se que seja favorecido pelo 'vento iônico' que acaba por acentuar seu efeito fazendo com que a força resultante exceda o seu peso.

Existem inúmeras patentes sobre métodos para gerar propulsão utilizando-se tais capacitores, que se atribuem ao efeito Biefeld-Brown. O próprio Brown patenteou sua descoberta ('aparato eletrocinético') nos anos de 1960, 1962 e 1965. Entretanto a descoberta de Brown não teve maior repercussão e praticamente pouco se fez até o final do século XX. A partir do ano 2000 houve uma 'redescoberta' do fenômeno e atualmente existem inúmeras demonstrações deste efeito na internet [1] em capacitores assimétricos denominados 'lifters' ('levitadores'). Há uma grande quantidade de informação, porém nos textos encontrados na rede de computadores é usual encontrarmos expressões como 'antigravidade' e 'eletrogravidade' para tentar explicar 
o fenômeno e que são estranhas à física tal como estabelecida atualmente. Praticamente nada se encontra sobre capacitores assimétricos nas revistas especializadas de física.

O propósito deste trabalho foi investigar o fenômeno usando equipamentos de construção própria, submeter o capacitor a diferentes tensões, variar os parâmetros geométricos e as características físicas a fim de se propor uma lei empírica da força atuante neste tipo de capacitor em atmosfera terrestre. Assim, este trabalho é uma tentativa de trazer algum entendimento, com base em dados experimentais, sobre as forças existentes em capacitores assimétricos. Este é um trabalho preliminar onde a abordagem, do ponto de vista teórico, é bastante simplificada face à complexidade do problema em questão, pois o campo elétrico entre as placas do capacitor tem intensidade suficiente para ionizar as moléculas do ar em sua volta.

\section{Construção do aparato}

\subsection{O tipo de capacitor utilizado}

Os capacitores utilizados são do tipo triangular (triângulo eqüilátero), construídos com bambu, papel alumínio, canudos plásticos e fio esmaltado com $0,1 \mathrm{~mm}$ de diâmetro. Na Fig. 1 mostramos o capacitor e suas principais dimensões.

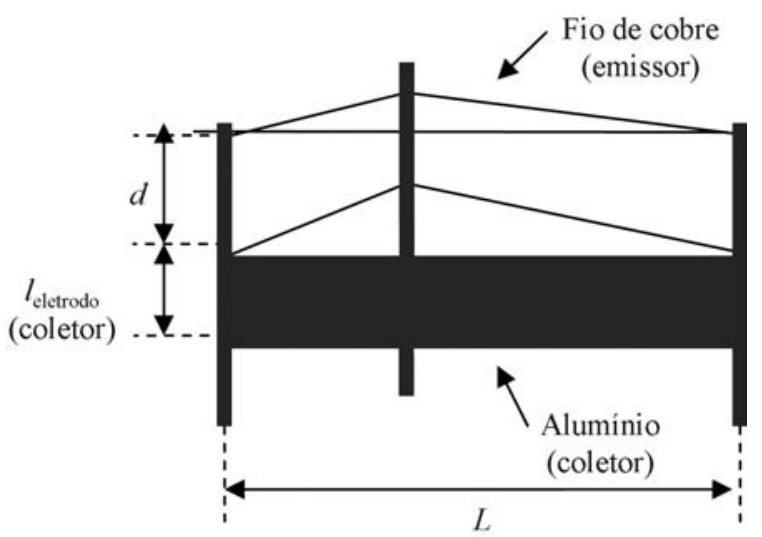

Figura 1 - Desenho do capacitor assimétrico de geometria triangular mostrando o eletrodo coletor (folha de alumínio), o eletrodo emissor (fio de cobre), a separação entre os eletrodos $d$, e a largura do coletor $l_{\text {eletrodo. }}$

O eletrodo superior consiste de um fio e o inferior de três placas feitas com papel alumínio. Como se vê, as características dos eletrodos o definem como assimétrico. Para a realização dos experimentos foram construídos capacitores de mesma geometria (triangular), porém com eletrodos de comprimentos $L=5,10$, $15,20,25,30,40$ e $50 \mathrm{~cm}$.

\subsection{Fonte de alimentação utilizada}

Para se obter grandes diferenças de potencial $(\sim 85 \mathrm{kV}$ DC) foi utilizado um sistema inversor transistorizado de alta freqüência ( $\sim 15 \mathrm{kHz})$ cujo sinal é aplicado a uma bobina enrolada manualmente sobre um transformador de saída vertical de vídeo, gerando tensões de saída da ordem de $30 \mathrm{kV}$ AC. Acoplando agora um triplicadorretificador (TRIPLER) na saída do fly-back atingimos tensões DC elevadas, da ordem de aproximadamente $85 \mathrm{kV}$ DC.

Variando a tensão de entrada (de zero a $250 \mathrm{~V} \mathrm{AC}$ ) por meio de um varivolt linear (transformador-variador de tensão), podemos controlar a tensão de saída da fonte de baixa tensão (que alimenta o inversor) entre zero e $50 \mathrm{~V}$ (DC) o que permite variarmos a alta tensão entre 0 e $85 \mathrm{kV}$. Para determinar os valores da alta tensão, utilizamos o "método do faíscamento", cujas medidas coincidem com as encontradas na literatura [2]. Sabendo que a tensão de ruptura da rigidez dielétrica do ar é de aproximadamente $1000 \mathrm{~V} / \mathrm{mm}$ (umidade relativa média de 50\%), Ref. [2-4], podemos medir o tamanho da centelha produzida pela alta tensão e assim relacioná-la com a tensão de entrada, de fácil medição. Os resultados das medidas estão na Fig. 2 onde se mostra a tensão do secundário (alta tensão) versus tensão do primário. Os resultados podem ser adequadamente descritos em termos de uma relação linear do tipo $V_{s}=A+B V_{p}$ onde $V_{s}$ é a tensão do secundário (em $\mathrm{kV}$ ) e $V_{p}$ é a tensão do primário (em Volts). Os valores obtidos para as constantes A e B são, respectivamente, $-17( \pm 2)$ e $0,411( \pm 0,007)$. Existe uma imprecisão inerente ao tipo de medida realizada e, como em geral, a tensão de ruptura da rigidez dielétrica do ar pode variar até $20 \%$ (dependendo da temperatura e umidade relativa do ar, para o ar seco $\sim 3 \mathrm{kV} / \mathrm{mm}$ ) $[2,4]$, podemos considerar que as tensões do secundário estão dentro desta ordem de precisão.

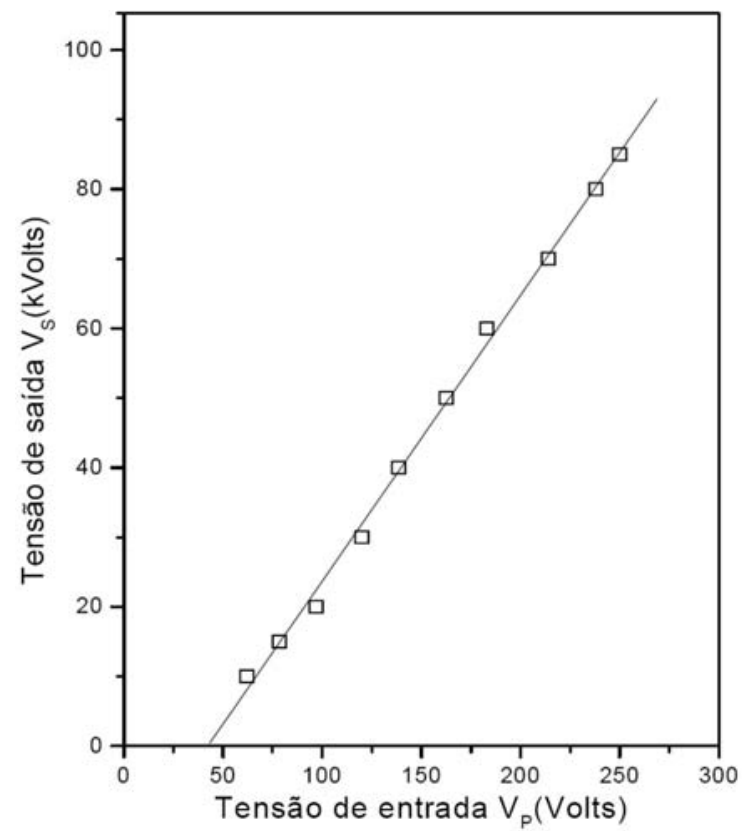

Figura 2 - Gráfico da tensão no secundário $V_{s}$ em função da tensão de entrada $V_{p}$. 


\section{Sistema de medida estática da força que atua no capacitor}

\subsection{Balança de mola}

Um sistema de medida eficiente, adotado para medir a força, consiste em acoplar o capacitor a um dinamômetro muito sensível e adicionar uma massa $m$ ao sistema, já que a força pode exceder o peso $m_{c} \boldsymbol{g}$ do capacitor, e assim, medir o deslocamento da mola.

Como as forças envolvidas são pequenas, foi utilizada uma mola de constante elástica muito pequena $(k=0,239 \mathrm{~N} / \mathrm{m})$. A Fig. 3 ilustra o dispositivo utilizado.

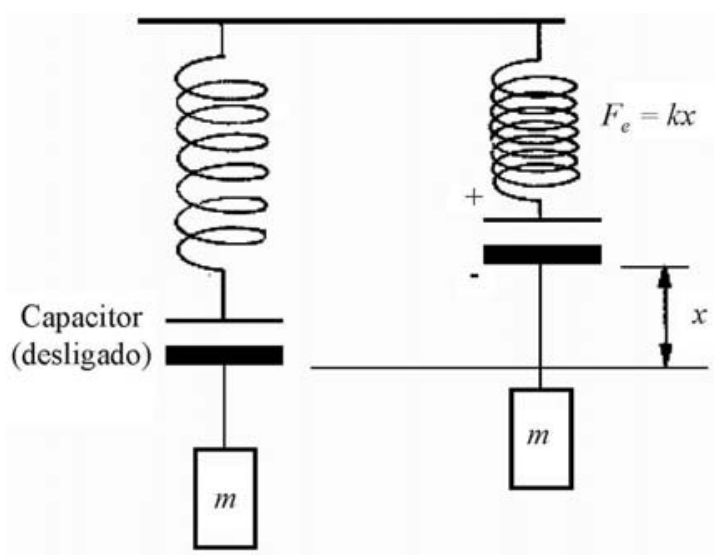

Figura 3 - Diagrama esquemático mostrando o sistema utilizado para medir a força sobre o capacitor assimétrico.

A força sobre o capacitor, $\mathbf{F}$, é diretamente proporcional ao deslocamento da mola. O peso $\left(m_{c}+m\right) \boldsymbol{g}$ deve ser sempre maior do que o empuxo máximo do capacitor para que o mesmo não flutue, impossibilitando qualquer medida.

\section{Resultados e discussão}

Os capacitores assimétricos são caracterizados pelo seu lado $L$, pela largura do eletrodo inferior $l_{\text {eletrodo }}$ (ou coletor), pela distância $d$ (entre os eletrodos) e pelo fio, que é o eletrodo superior (ou emissor), conforme mostrado na Fig. 1. Inicialmente investigamos o comportamento da força sobre o capacitor considerando a variação da largura do eletrodo coletor. Escolhemos $L=20 \mathrm{~cm} \mathrm{e} d=6 \mathrm{~cm}$ e aplicamos uma tensão de $55 \mathrm{kV}$. Na Fig. 4 apresentamos os resultados obtidos para a força vs. $l_{\text {eletrodo }}$.

Podemos observar que a força é nula para capacitores simétricos, crescendo rapidamente à medida que cresce a largura do coletor, tendendo a um valor limite, isto é, tornando-se independente da largura do coletor para valores de $l_{\text {eletrodo }} \geqslant 3 \mathrm{~cm}$. Devido a este comportamento da força nos demais experimentos consideramos $l_{\text {eletrodo }}$ fixo e igual a $3 \mathrm{~cm}$.

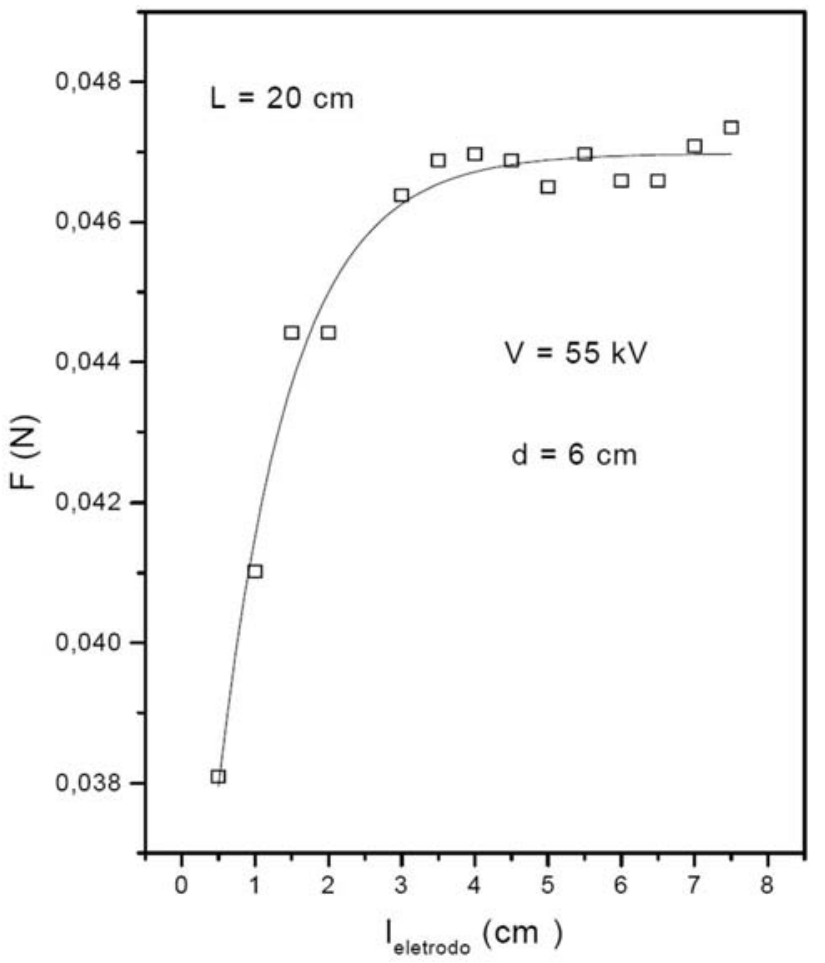

Figura 4 - Gráfico da força em função da largura do eletrodo. Distância entre eletrodos $d=6 \mathrm{~cm}, \vec{L}=20 \mathrm{~cm}$ e tensão aplicada de $55 \mathrm{kV}$.

Em uma segunda etapa verificamos o comportamento da força em relação ao lado $L$ dos capacitores. Neste caso consideramos primeiro $d=6 \mathrm{~cm}$, e aplicamos uma tensão de $55 \mathrm{kV}$. Em seguida aumentamos $d$ para $10 \mathrm{~cm}$ e aplicamos uma tensão de $85 \mathrm{kV}$. Os resultados estão na Fig. 5.

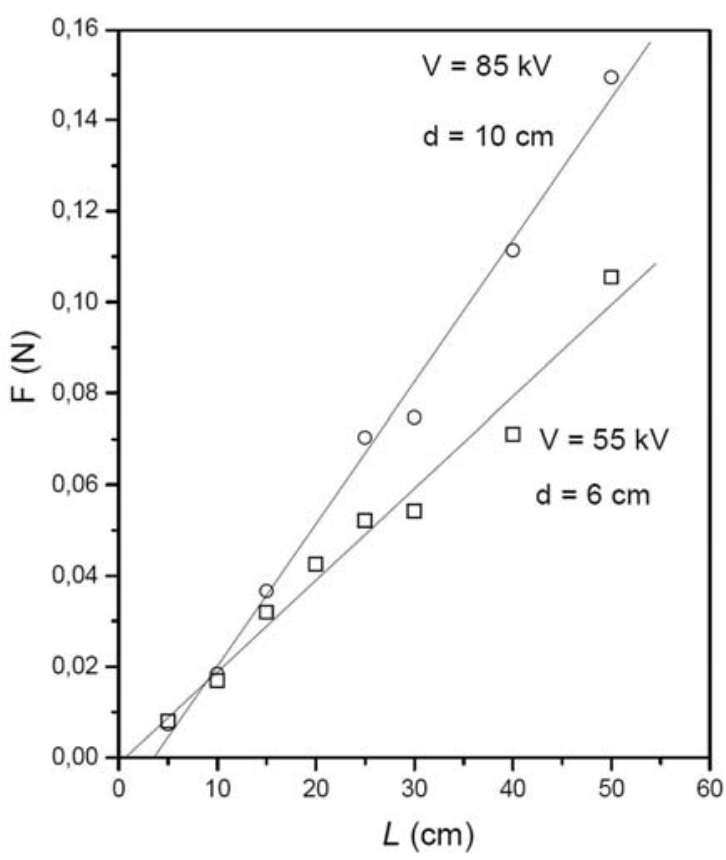

Figura 5 - Força sobre o capacitor assimétrico em função do comprimento $L$ do coletor, para dois diferentes valores de $V$ e $d$. 
Os resultados mostram uma variação linear da força com o lado do capacitor e esta dependência pode ser entendida lembrando que o volume de gás passível de ionização em um cilindro de raio $R$ em torno do eletrodo emissor cresce com $\pi R^{2} L$. Assim, quanto maior o valor de $L$ do emissor, maior é o número de moléculas que são ionizadas em torno do fio, aumentando o fluxo de íons. Podemos observar também que existe uma dependência da força sobre o capacitor com a tensão aplicada e a distância $d$ entre os eletrodos. A Fig. 5 mostra que a força cresce linearmente com a tensão apesar do aumento da distância $d$ entre os eletrodos, de $6 \mathrm{~cm}$ $(V=55 \mathrm{kV})$ para $10 \mathrm{~cm}(V=85 \mathrm{kV})$. Portanto, fica clara a necessidade de conhecermos como a força varia com $d$ e com a tensão aplicada. Devemos lembrar que a tensão máxima que podemos aplicar depende da rigidez dielétrica do ar. Nas condições normais de temperatura e pressão resultados experimentais demonstram que temos por volta de $1 \mathrm{kV}$ por milímetro. Logo para uma distância $d=3 \mathrm{~cm}$, pode-se aplicar no máximo $30 \mathrm{kV}$, para $d=6 \mathrm{~cm}$, podemos aplicar no máximo $60 \mathrm{kV}$, sem que ocorra descarga entre os eletrodos.

A dependência da força com a distância $d$ entre os eletrodos foi verificada considerando um capacitor de $L=15 \mathrm{~cm}$ e onde aplicamos, para cada valor de $d$, tensões de $55 \mathrm{kV}$ e $85 \mathrm{kV}$. Na Fig. 6 mostramos a força em função da distância $d$ entre os eletrodos.

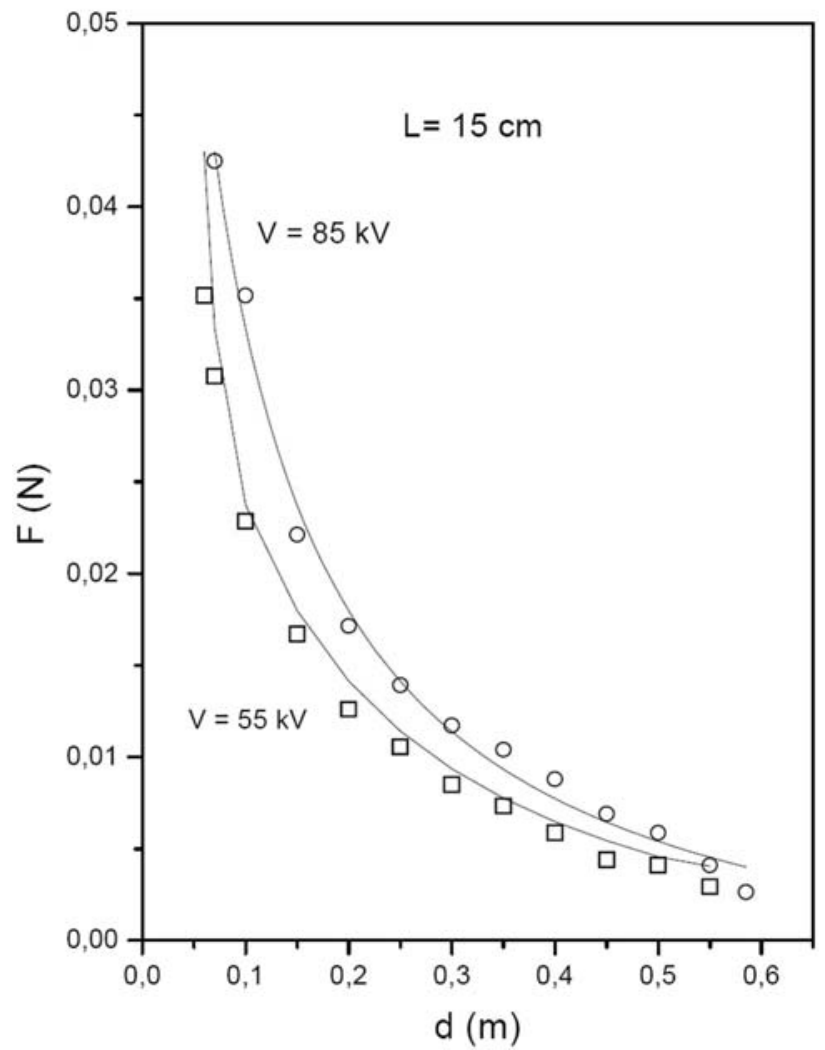

Figura 6 - Força sobre o capacitor assimétrico em função da separação entre os eletrodos para dois valores de tensão aplicada.

Como podemos observar, a força cai rapidamente com o aumento da distância entre os eletrodos. Isto está diretamente ligado à variação espacial do campo elétrico. Dos resultados mostrados na Fig. 6 temos $F$ variando com $1 / d^{\beta}$. Na Fig. 7 mostramos a força em função do inverso da distância.

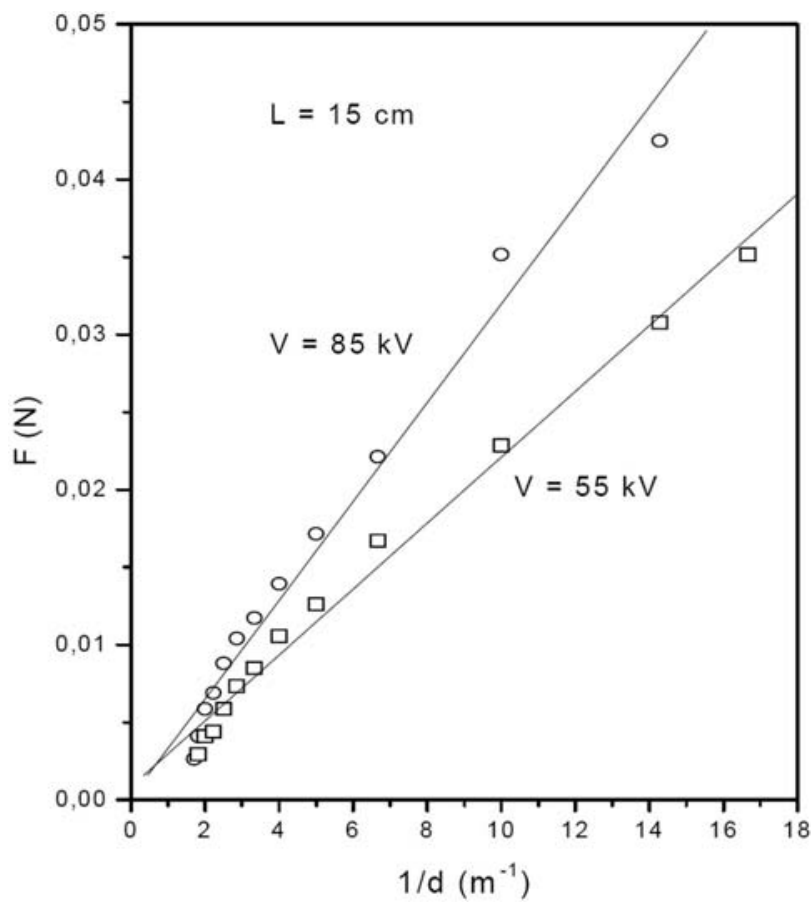

Figura 7 - Força sobre o capacitor assimétrico em função do inverso da separação entre os eletrodos para dois valores de tensão aplicada.

Podemos ver que uma função linear se ajusta bem aos resultados obtidos, mostrando que $\beta=1$. Podemos inferir facilmente que este comportamento da força está relacionado à variação espacial do campo elétrico, que para um fio carregado varia com $1 / r$, [3, 4]. É importante lembrar aqui que este comportamento da força com $d$, isto é, variando com o inverso da distância, foi obtido para um dado valor de largura do eletrodo coletor, $l_{\text {eletrodo. Uma investigação mais detalhada requer }}$ a variação de $l_{\text {eletrodo, }}$ considerando-se diferentes valores de $L$, e para cada valor de $L$, considerarmos a variação de $l_{\text {eletrodo, }}$ para diferentes valores de $d$. Por exemplo, como mostrado na Fig. 4, o comportamento

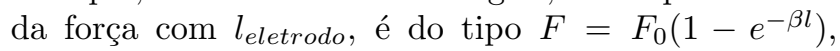
onde $l=l_{\text {eletrodo. }}$ Considerando que obtivemos o comportamento da força como sendo proporcional a $1 / d$, podemos inferir um comportamento do tipo $F=$ $F_{0}\left(1-e^{-\alpha l / d}\right) / d$, onde $F_{0}$ e $\alpha$ devem ser determinados experimentalmente. Neste trabalho não levamos em conta este fato e consideramos uma abordagem mais simples onde a força é uma função apenas do inverso da distância.

É fácil perceber, por meio dos resultados acima apresentados, que a força cresce com a tensão aplicada no capacitor. Efetuamos medidas da força variando-se a tensão aplicada para capacitores assimétricos de lados 
$L=15,25,30,40$ e $50 \mathrm{~cm}$. Os resultados obtidos estão apresentados nos gráficos mostrados na Fig. 8 (gráficos (a), (b), (c) e (d)). Foram considerados dois valores de $d$ (distância entre eletrodos) para os capacitores com lados de 15,25 e $50 \mathrm{~cm}$.
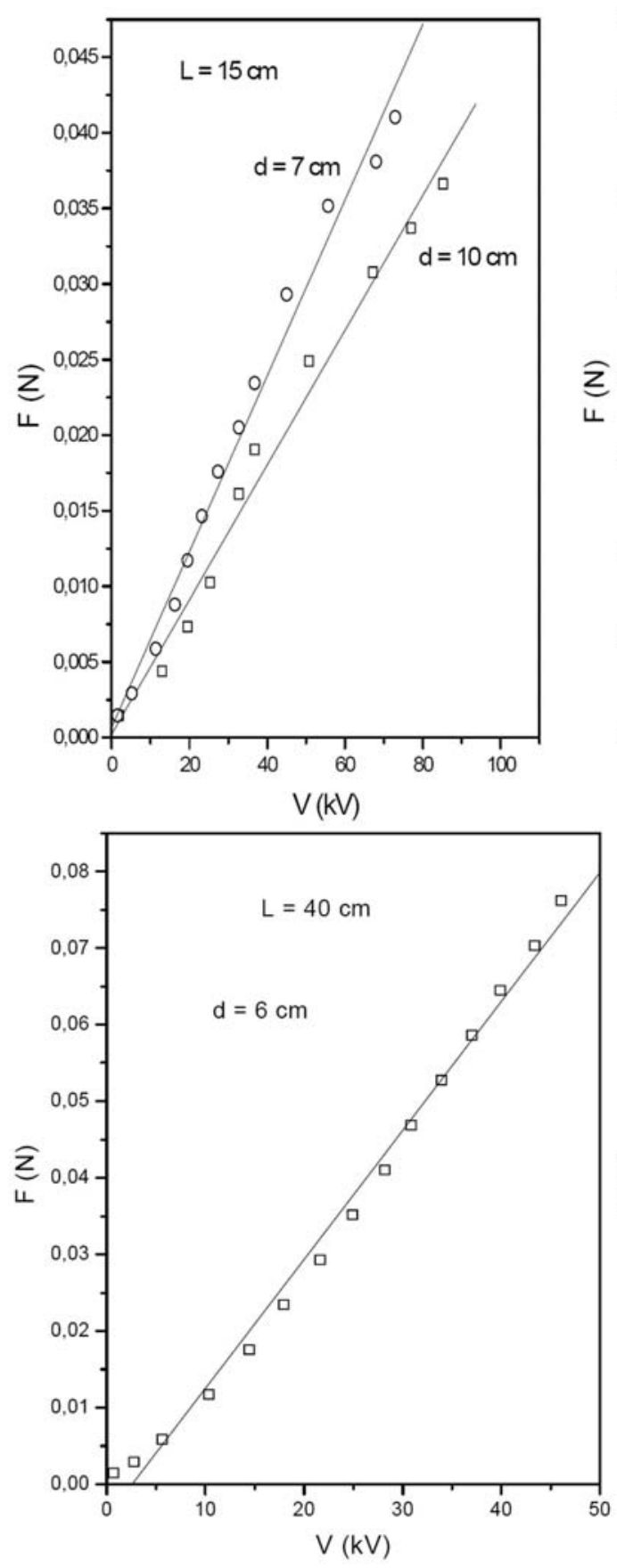
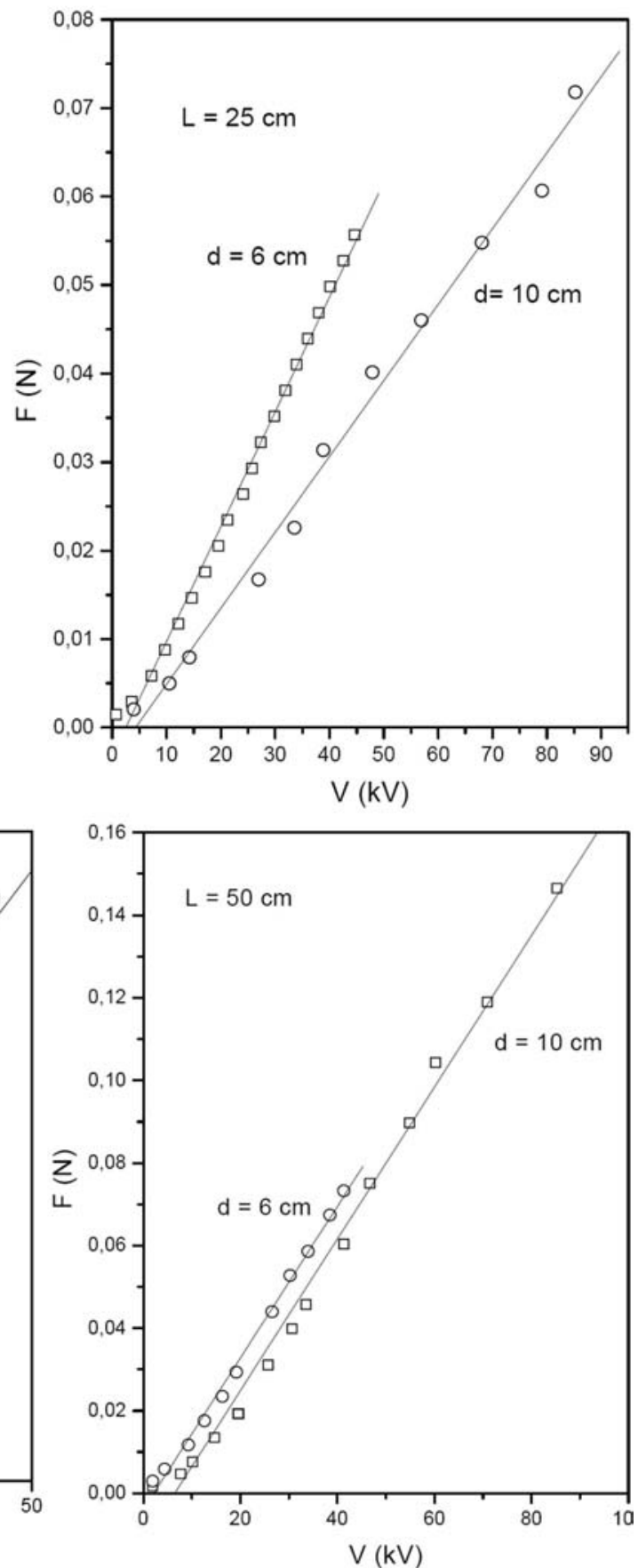

Figura 8 - Forças sobre o capacitor assimétrico em função da tensão aplicada para diferentes valores de L e $d$.

Dentro dos limites de precisão das medidas de força e tensão efetuadas, podemos ver que, a partir dos resultados mostrados na Fig. 8, existe uma relação linear entre a força e a tensão aplicada no capacitor. A partir dos resultados mostrados nos gráficos da Fig. 8, percebe-se a existência de uma tensão de corte $\left(V_{c}\right)$ e que não está bem definida, decorrente das imprecisões nas medidas de força (bem pequenas) para baixas tensões (entre 1 e $5 \mathrm{kV}$ ). A existência de uma tensão de corte indica a necessidade de uma tensão mínima 
para o aparecimento de uma força resultante sobre o capacitor. Para avançarmos na compreensão da força sobre o capacitor, lembramos que se ela é proporcional a $L$, a $V$, e inversamente proporcional a $d$, então é proporcional ao produto destas variáveis. Na Fig. 9 apresentamos os resultados para a força como função do produto $L V / d$.

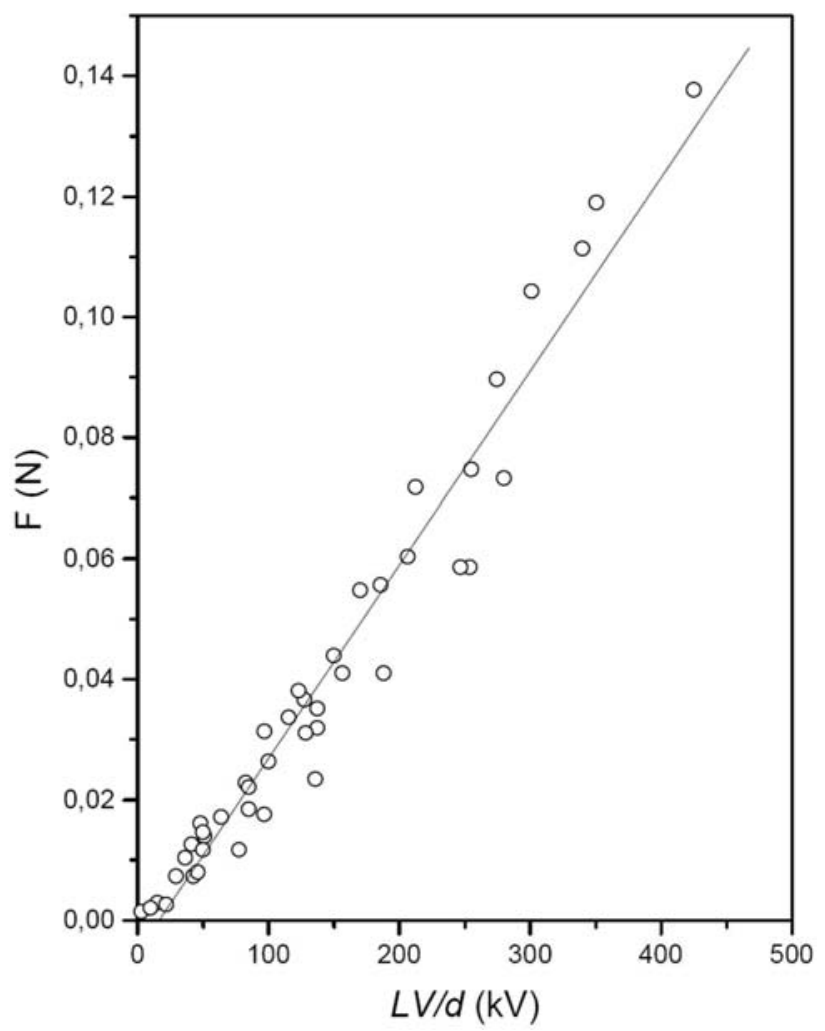

Figura 9 - Força sobre o capacitor assimétrico em função do produto $L V / d$.

É fácil constatar que temos um certo espalhamento nos resultados, conseqüência das imprecisões nas medidas efetuadas. Considerando um ajuste linear, obtemos para a força

$$
F=k L V / d+k_{0},
$$

onde $k=3,2 \times 10^{-4} \mathrm{~N} / \mathrm{kV}\left( \pm 0,1 \times 10^{-4}\right)$ e $k_{0}=-0,0053$ $\mathrm{N}( \pm 0,0005)$. Os valores de $L$ e $d$ devem ser dados em metros e a tensão em kV. Obviamente podemos reescrever a Eq. (1) como

$$
F=k \frac{L}{d}\left(V-V_{1}\right),
$$

onde $V_{1}=\left(\left|k_{0}\right| / k\right) d / L=V_{c} d / L$ onde $V_{c}=16,45 \mathrm{kV}$ é a tensão de corte e que depende do meio no qual o capacitor está (o dielétrico) e também do diâmetro do fio utilizado como eletrodo emissor (no caso, 0,1 mm). As medidas de força foram realizadas nas condições normais de temperatura e pressão com uma umidade relativa média de $50 \%$. É certo que as constantes $k$ e $k_{0}$ dependem do meio em que se encontra o capacitor. Isto é, as constantes $k$ e $k_{0}$ (e a tensão de corte), dependem da densidade do meio, da temperatura e da umidade relativa. Efetuamos experiências qualitativas com o capacitor em ambiente fechado, onde elevamos a umidade relativa do ar para valores acima de $70 \%$ e verificamos uma redução drástica na força sobre o capacitor. Entretanto, até o momento, não efetuamos medidas quantitativas da força considerando-se variações na umidade relativa do ar.

A partir dos resultados para as forças em capacitores assimétricos, mostrados nas Figs. 5 a 8, percebe-se que as forças são de baixa intensidade, sempre da ordem de gramas-força. Naturalmente isto se reflete no pequeno valor da constante $k$ na Eq. (2) de onde podemos notar que para conseguirmos forças apreciáveis, os capacitores devem ter dimensões consideráveis. Por exemplo, se temos um capacitor de lado igual a dez metros e se aplicarmos $300 \mathrm{kV}$, tem-se uma distância $d$ mínima de $30 \mathrm{~cm}$, e assim a força sobre este capacitor será igual a 3,20 N, ou seja, irrisória. Assim, é possível concluir que a utilização destas forças em sistemas de propulsão encontra um sério fator limitante, que é justamente a intensidade das forças envolvidas. Neste momento também é oportuno lembrar que, aparentemente, não consideramos a dependência da força com o diâmetro do fio utilizado (eletrodo emissor). Mas isto é fácil de entender. Se aumentarmos o diâmetro do fio, diminuímos a densidade de carga (a um mesmo potencial) e logo diminuímos a intensidade do campo, que é proporcional à densidade de carga. Portanto a força necessariamente decresce, isto é, quando aumentamos o diâmetro do fio, estamos tornando o capacitor cada vez mais simétrico, fazendo com que a força diminua progressivamente.

Em relação à variação da densidade do meio em que se encontra o capacitor, faremos algumas considerações mais adiante. Vamos primeiro considerar a relação entre a força sobre o capacitor expressa empiricamente pela Eq. (2) acima e o meio circundante, o ar, que consideramos, por simplicidade, um gás de $\mathrm{N}_{2}$. Esta simplificação não elimina, de fato, a presença de moléculas de $\mathrm{O}_{2}$ e $\mathrm{H}_{2} \mathrm{O}$, que explicam a forte produção de ozônio $\left(\mathrm{O}_{3}\right)$ quando aplicamos uma tensão $\mathrm{V}$ nos capacitores. Devido ao campo elétrico muito intenso, ocorre a quebra de moléculas de água, liberando oxigênio atômico, que reage com as moléculas de oxigênio produzindo ozônio. Lembramos que as energias de ligação das moléculas de $\mathrm{N}_{2}$ e $\mathrm{O}_{2}$ são elevadas [5], isto é, $942 \mathrm{~kJ} / \mathrm{mol}$ e $494 \mathrm{~kJ} / \mathrm{mol}$ respectivamente, enquanto que para a água temos $200 \mathrm{~kJ} / \mathrm{mol}$. Portanto, dissociamos moléculas de água mais facilmente. Na experiência relacionada com a umidade relativa, já comentada acima, observou-se que no transcorrer de dois minutos em que o capacitor ficou em operação (tensão de $85 \mathrm{kV}$ ) a umidade relativa do ar na câmara baixou em torno de $5 \%$, o que indica a dissociação de um grande número de moléculas de água do meio. 
Se admitirmos que os capacitores estejam imersos em um gás de $\mathrm{N}_{2}$ deve-se considerar a ionização destas moléculas pelo campo elétrico intenso existente entre as placas do capacitor e a velocidade de arraste dos íons $\mathrm{N}_{2}^{+}$produzidos. Especificamos esta velocidade de arraste pela mobilidade $\mu_{\text {íon }}$ dos íons a qual descreve a migração destes na presença de um campo elétrico em um dado meio. A velocidade de arraste dos íons é $\boldsymbol{v}_{\text {íon }}=\mu_{\text {íon }} \mathbf{E}$, onde $\mathbf{E}$ é o campo elétrico aplicado e o valor médio ${ }^{6}$ da mobilidade $\left(\mu_{\text {íon }}\right)$ de íons positivos no ar $\left(\mathrm{N}_{2}^{+}\right)$é $\mu_{\text {íon }}=1,4 \times 10^{-4} \mathrm{~m}^{2} / \mathrm{Vs}$. Os íons produzidos transportam uma quantidade de movimento e podemos estimar o número de íons por segundo $\left(n_{\text {íon }}\right)$ necessários para que o momento transportado por eles se equipare à força sobre o capacitor. Assim, a força correspondente a este fluxo de íons é dada por

$$
F=n_{\text {ion }} m_{N_{2}} v_{\text {íon }} \text {. }
$$

Considerando por simplicidade, $v_{\text {ín }}=\mu_{\text {ion }} V / d$, (numa aproximação em que o campo é tratado como uniforme) e igualando-se as Eqs. (2) e (3) obtemos para o número $n_{\text {íon }}$ de íons por segundo

$$
n_{\text {ion }}=\frac{k L}{m_{N_{2}} \mu_{i o n}}\left(\frac{V-V_{1}}{V}\right) .
$$

Observamos, como esperado, que o número de íons produzidos pelo campo cresce com $L$ e sua dependência com a distância $d$ (entre eletrodos) está implícita em $V_{1}$. Para um capacitor com lado igual a $50 \mathrm{~cm}$ e $d=10 \mathrm{~cm}$, tem-se $V_{1}=3,3 \mathrm{kV}$ e para uma tensão aplicada de $80 \mathrm{kV}$ o fluxo de íons será $n_{\text {íon }} \cong 5 \times 10^{25}$ íons/s. Para um capacitor de lado igual $5 \mathrm{~cm}$ e $d=10 \mathrm{~cm}$ temos $V_{1}=32,8 \mathrm{kV}$ e, aplicando-se uma tensão de $80 \mathrm{kV}$, obtemos $n_{\text {íon }} \cong 3 \times 10^{24}$ íons $/ \mathrm{s}$. Um número tão elevado de íons por segundo representa uma corrente extraordinariamente alta, o que não é verificado na prática. As correntes medidas nos capacitores raramente excedem a 1mA! Além disso, as velocidades destes íons são bastante elevadas, pois para $V=80 \mathrm{kV}$ e $d=10 \mathrm{~cm}$ temos $v_{\text {ion }} \cong 110 \mathrm{~m} / \mathrm{s}$, que é um valor muito elevado para velocidade dos íons e que não corresponde à velocidade de deslocamento do ar que podemos sentir ao colocarmos a mão sob o capacitor enquanto ele flutua, que é bastante pequena.

Isto significa que devemos levar em conta que os íons produzidos pelo campo colidem com as moléculas de $\mathrm{N}_{2}$ transferindo momento a elas. Assim temos um fluxo final de moléculas do gás transportando uma quantidade de movimento numa direção que equilibra o momento adquirido pelo capacitor no sentido oposto, explicando as pequenas correntes que são medidas nos capacitores quando em operação. A enorme quantidade de íons produzidos próximos a um eletrodo não alcança o outro, devido às colisões com as moléculas do meio. Próximo ao fio, onde o campo é muito intenso, a produção de íons certamente ocorre por um processo de avalanche, não ocorrendo o mesmo para pontos mais afastados do fio. Portanto esta grande quantidade de íons colide com as moléculas da vizinhança gerando, dessa maneira, um fluxo do gás. Para um gás com densidade $\rho$ que flui através de uma área $A$, com velocidade de arraste $v_{\rho}$, temos uma força

$$
F=\rho A v_{\rho}^{2}
$$

sendo $A$ a área do capacitor, um triângulo eqüilátero de lado $L$, cuja área é igual a $(\sqrt{3} / 4) L^{2}$. Como esta força deve ser igual à força sobre o capacitor dada pela Eq. (2), obtemos para a velocidade de arraste das moléculas do meio

$$
v_{\rho}=\sqrt{\frac{4 k\left(V-V_{1}\right)}{\sqrt{3} \rho L d}} .
$$

Para um capacitor de lado $L=50 \mathrm{~cm} \mathrm{e} d=6 \mathrm{~cm}$, vem $V_{1}=1,97 \mathrm{kV}$ e para $V=60 \mathrm{kV}$ obtemos $v_{\rho} \cong 1,30 \mathrm{~m} / \mathrm{s}$, onde usamos $0,85 \mathrm{~kg} / \mathrm{m}^{3}$ para a densidade do ar. Para um capacitor de lado $L=5 \mathrm{~cm}$, com $d=10 \mathrm{~cm}$ e aplicando-se uma tensão $V=80 \mathrm{kV}$, tem-se $v_{\rho} \cong 0,90 \mathrm{~m} / \mathrm{s}$. Estes valores para a velocidade das moléculas do gás correspondem ao leve vento que podemos sentir ao colocarmos a mão estendida sob o capacitor quando em operação. Estes resultados indicam que a força atuante sobre o capacitor está diretamente relacionada ao movimento adquirido pelas moléculas do gás que constituem o meio no qual está imerso o capacitor devido às colisões destas com íons produzidos pelo campo elétrico e que são acelerados por este campo.

Podemos obter o número de moléculas $(n)$ por segundo, de massa $m_{N_{2}}$, com velocidade $v_{\rho}$, igualando a força correspondente, isto é

$$
F=n m_{N_{2}} v_{\rho}
$$

à força dada pela Eq. (2), e usando $v_{\rho}$ dado pela Eq. (6). Com este procedimento obtemos para $n$

$$
n=\frac{\left.\sqrt{k L \rho A\left(\frac{V-V_{1}}{d}\right.},\right)}{m_{N_{2}}}
$$

onde $A$ é área do capacitor. Para um capacitor com $L=50 \mathrm{~cm} \mathrm{e} d=6 \mathrm{~cm}$ aplicando-se uma tensão de $60 \mathrm{kV}$ obtemos $n \cong 5,3 \times 10^{24}$ moléculas de $\mathrm{N}_{2}$ por segundo deslocando-se com uma velocidade de $1,30 \mathrm{~m} / \mathrm{s}$, o que corresponde a quase 9 moles, significando assim uma massa total de $\mathrm{N}_{2}$ de apenas 0,118 gramas. Já para o capacitor cujo lado é $5 \mathrm{~cm}$ e com $d=10 \mathrm{~cm}$, aplicando uma tensão de $80 \mathrm{kV}$, vem $n \cong 5 \times 10^{23}$ moléculas de $\mathrm{N}_{2}$ por segundo deslocando com uma velocidade de $0,90 \mathrm{~m} / \mathrm{s}$. Portanto temos resultados perfeitamente plausíveis no que diz respeito ao que é observado, ou seja, o leve deslocamento de ar perceptível ao estendermos a mão sob o capacitor em operação. Não efetuamos medidas da velocidade deste vento, o que deve ser feito em uma investigação mais detalhada das 
forças em capacitores assimétricos, incluindo-se as variações da umidade relativa do ar, o tipo e a densidade do gás nos quais estejam imersos estes capacitores.

Portanto, em capacitores assimétricos submetidos a tensões elevadas o campo produz íons (que geram íons num efeito cascata) os quais transferem o momento linear adquirido pela ação do campo sobre os mesmos, às moléculas do ar. Isto gera um fluxo, cuja quantidade de movimento total deve ser a mesma que a do capacitor, que é o lugar onde residem as cargas-fonte do campo. Isto significa claramente que admitimos a conservação do momento linear durante o processo, ou seja, o momento linear dos íons (que é transferido para as moléculas do meio) igual ao momento das cargas-fonte (localizadas no capacitor). É fácil perceber que a etapa crucial é a produção de íons pelo campo e, como vimos, temos uma tensão de corte para que uma força surja sobre o capacitor. É necessário assim que tenhamos um campo que ionize as moléculas do meio na região próxima ao eletrodo emissor.

A partir dos resultados que obtivemos, nosso entendimento é que, para que o capacitor fique sujeito a uma força, é necessária a existência de um meio passível de ionização. Ou seja, a constante $k$ na expressão de força, dada pela Eq. (2), depende da densidade do meio, indo a zero quando a densidade vai a zero. Além disto, esta constante deve também depender do tipo de gás do qual o meio é constituído, pois cada gás tem seu potencial de ionização. Assim para um gás de baixo potencial de ionização haverá, para uma dada tensão, um maior número de íons produzidos do que para um gás com um potencial de ionização mais alto.

Em relação à densidade zero, ou seja, no vácuo, esperamos que não surja nenhuma força sobre o capacitor, já que não ocorre a produção de íons devido à inexistência de moléculas. Mas vamos admitir que a constante $k$, ao invés de ir a zero para densidade nula, vá para um valor diferente de zero. Assim, em um experimento com o capacitor na câmara de vácuo, teríamos apenas deslocamento de elétrons. Vamos considerar uma abordagem simples e admitir que o momento adquirido pelos elétrons, devido à presença do campo, seja transmitido ao capacitor, quando da colisão destes elétrons com o fio do capacitor. Como se pode notar, a idéia é que os elétrons recebem quantidade de movimento cedido pelo campo, que é estabelecido por uma fonte externa de energia, e a transferem ao capacitor e, assim, a energia do campo é convertida em mecânica (movimento do capacitor). Como as tensões aplicadas são bastante elevadas, os elétrons adquirem velocidades relativísticas. Supondo que a energia $e V$ seja convertida em energia cinética os elétrons adquirem uma velocidade igual a

$$
v=c \sqrt{1-\frac{1}{\left(1+\frac{e V}{m_{0} c^{2}}\right)^{2}}},
$$

onde $m_{0} c^{2}$ é a energia de repouso do elétron

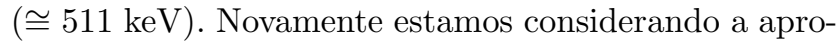
ximação mais simples em que o campo é tratado como uniforme. Para uma tensão aplicada de $80 \mathrm{kV}$, obtemos $v \cong 0,50 c$, indicando que realmente os elétrons alcançam velocidades extremamente elevadas (no vácuo). O tempo de vôo dos elétrons (de uma placa do capacitor a outra) é extremamente curto. Por exemplo, na situação mais simples de um movimento retilíneo, o tempo para o deslocamento é

$$
t=\frac{d}{c} \frac{m_{0} c^{2}}{e V} \sqrt{\left(1+\frac{e V}{m_{0} c^{2}}\right)^{2}-1}
$$

onde $d$ é a distância entre as placas e $V$ a tensão aplicada. Assim, para $d=10 \mathrm{~cm}$ a $30 \mathrm{kV}$, o tempo de deslocamento é 1,97 ns e para uma tensão de $80 \mathrm{kV}$ seu valor cai para 1,24 ns. Considerando que temos $n_{e}$ elétrons por segundo, a força transmitida ao capacitor é

$$
F=n_{e} m v=n_{e} \frac{m_{0}}{\sqrt{1-\frac{v^{2}}{c^{2}}}} v
$$

Admitindo-se a validade da Eq. (2), obtemos para $n_{e}$

$$
n_{e}=\frac{k L\left(V-V_{1}\right)}{m_{0} c d} \frac{1}{\sqrt{\left(1+\frac{e V}{m_{0} c^{2}}\right)^{2}-1}}
$$

onde usamos a Eq. (9) para $v$. Considerando-se os exemplos anteriores, isto é, um capacitor de lado de $50 \mathrm{~cm}$ e com $d=10 \mathrm{~cm}$ a $80 \mathrm{kV}$ a Eq. (12) fornece $n_{e}=7,75 \times 10^{20}$ elétrons por segundo, o que significa uma corrente de $124 \mathrm{~A}$ ! (para gerar uma força sobre o capacitor de apenas 0,12 N!) É fácil perceber que não temos uma corrente desta ordem de grandeza. Muito pelo contrário espera-se que a corrente no capacitor seja, como no caso de pressão ambiente, da ordem de miliampéres. Para obtermos correntes desta ordem de grandeza, utilizando a Eq. (12), vemos que é necessária a redução da constante de força $k$ por um fator da ordem de $10^{4}$ a $10^{5}$. Ou seja, ao invés de ir a zero para densidade nula do gás, a constante $k$, no vácuo, teria um valor até cem mil vezes menor do que seu valor nas condições normais de operação (1 atm) e, conseqüentemente, teríamos forças até cem mil vezes menores atuando nos capacitores! Forças de dificílima medição! É nosso ponto de vista que esta questão permanece em aberto, enquanto medidas variando a densidade do meio, até se atingir alto vácuo, não forem efetuadas. Acreditamos que estas medidas devam ser feitas com um grau de precisão bem mais elevado dos que as que foram realizadas no presente trabalho. 


\section{Conclusões}

Neste trabalho investigamos o comportamento da força em capacitores assimétricos e submetidos a tensões bastante elevadas. Dentro dos limites de precisão das medidas efetuadas, os resultados alcançados indicam uma variação linear da força com a tensão aplicada e com o lado do capacitor (ou seu perímetro) e variando com o inverso da distância entre os eletrodos. Os resultados mostram que as forças envolvidas são bastante pequenas, sendo este fato um fator limitante na utilização deste efeito em sistemas de propulsão.

Procurou-se estabelecer a origem da força partindose da formação de íons os quais ganham energia e momento do campo elétrico e transferem por meio de colisões às moléculas do gás circundante, produzindo assim um vento, que é perceptível quando o capacitor está em operação. O momento adquirido pelo total de moléculas do gás é igual (por conservação de momento) ao momento adquirido pelo lugar físico das cargas-fonte do campo, isto é, o capacitor. Mediante a produção de íons ocorre uma transformação de energia do campo em energia mecânica, correspondente ao deslocamento das moléculas do gás, produzindo um vento, e ao movimento do capacitor. Esta energia é oriunda de uma fonte externa de alimentação, que estabelece o campo. Nossos resultados indicam que se este efeito existe no vácuo (o que seria a contribuição eletrônica e que es- taria sempre presente) então as forças envolvidas são da ordem de dez mil a cem mil vezes menores que as existentes quando o capacitor está em operação nas condições normais de temperatura, pressão e umidade relativa do ar. Pensamos que as medidas efetuadas tenham lançado alguma luz sobre o fenômeno físico relacionado a forças em capacitores assimétricos e que para um entendimento mais profundo, primeiro são necessárias averiguações experimentais mais refinadas e precisas.

\section{Referências}

[1] http://jnaudim.free.fr/lifter/main.htm.

[2] A. von Engel, Ionized Gases (American Vacuum Society, editado pelo American Institute of Physics, Nova Iorque, 1994), p. 200.

[3] David Halliday e Robert Resncik, Física (Ed. Livros Técnicos e Científicos, S. Paulo, 1973), v. 2 p. 112.

[4] J.R. Reitz, F.J. Milford e R.W. Christy, Fundamentos da Teoria Eletromagnética (Ed. Campus, Rio de Janeiro, 1982), cap. 4 p. 12

[5] Donald A. McQuarrie e John D. Simon, Physical Chemistry - A Molecular Approach (University Science Books, Sausalito, 1997), cap. 9 e 10.

[6] Handbook of Chemistry and Physics $80^{\text {th }}$ (Taylor \& Francis CRC PRESS, Nova Iorque, Edition 1999-2000), 14-32. 